\title{
Medidas de avaliação de qualidade de vida e estados de saúde em ortopedia*
}

\author{
Quality of life and health status evaluation measurements
}

\author{
Andréa Diniz Lopes ${ }^{1}$, Rozana Mesouita Ciconelli2 ${ }^{2}$ Fernando Baldy dos Reis ${ }^{3}$
}

\section{RESUMO}

Medidas de qualidade de vida e estado de saúde têm sido enfatizados pela comunidade ortopédica por possibilitarem a análise da situação de saúde e as manifestações da doença na vida do indivíduo em sua própria perspectiva. Estudos têm analisado criteriosamente os instrumentos disponíveis e publicado recomendações direcionando a escolha dos mais adequados para 0 uso. $O$ presente estudo fornece descrição breve dos tipos de instrumentos, suas aplicabilidades, o processo de validação, os instru-

* Pesquisa realizada no Departamento de Ortopedia e Traumatologia da Escola Paulista de Medicina - Universidade Federal de São Paulo - UNIFESP - São Paulo (SP), Brasil.

1. Pós-graduanda do Departamento de Ortopedia e Traumatologia da Escola Paulista de Medicina - Universidade Federal de São Paulo - UNIFESP - São Paulo (SP), Brasil.

2. Professora Afiliada da Disciplina de Reumatologia do Departamento de Medicina da Escola Paulista de Medicina - Universidade Federal de São Paulo - UNIFESP - São Paulo (SP), Brasil.

3. Livre-docente, Professor Associado da Disciplina de Traumatologia do Departamento de Ortopedia e Traumatologia da Escola Paulista de Medicina - Universidade Federal de São Paulo UNIFESP - São Paulo (SP), Brasil.

Endereço para correspondência: Dr. Fernando Baldy dos Reis, Rua Adma Jafet, 50, 14o andar, Bela Vista - 01308-050 - São Paulo (SP), Brasil. Tel.: (11) 8326-7266.

Copyright RBO2007 mentos mais comumente utilizados na literatura internacional e aqueles disponíveis para uso no Brasil.
Descritores - Qualidade de vida; Questionários; Indicado- res básicos de saúde; Doenças músculo-esque- léticas/diagnóstico

\section{ABSTRACT}

Measurements of quality of life and health status have been emphasized by the orthopedic community because they allow for an analysis of the health status and disease manifestations according to the own point of view of the individual. Studies have carefully analyzed the tools available and published recommendations for the most adequate choice of such tools. This study provides a brief description of the types of instruments, their application, their validation procedure, the instruments that are more commonly seen in the international literature, and those available for use in Brazil.
Keywords - Quality of life; Questionaires; Health status indicators; Musculoskeletal diseases / diagnosis

\section{INTRODUÇÃO}

Nos últimos anos tem ocorrido mudança nos desfechos usados nas análises de efetividade de tratamentos clínicos ou cirúrgicos em ortopedia. Geralmente avaliavam-se as mudanças clínicas através do exame 
físico e exames complementares. Nas últimas décadas, desfechos como qualidade de vida relacionada a saúde, capacidade funcional, escalas de dor e satisfação têm sido enfatizados por possibilitarem a análise da situação de saúde e as manifestações da doença na vida do indivíduo em sua própria perspectiva (subjetividade), complementando os dados clínicos e objetivos. Como conseqüência, foram desenvolvidas e publicadas uma variedade de instrumentos, questionários e escalas que abordam esse tipo de variável, já que o exame objetivo é um indicador escasso para avaliação de aspectos funcionais, sociais e emocionais ${ }^{(1-4)}$.

O propósito desse interesse crescente da comunidade científica para o uso de tais instrumentos deriva de suas diversas aplicações, tais como percepção individual ou populacional do estado de saúde, avaliação de resultados e efetividade de tratamentos, possibilidade de uso em pesquisas clínicas e análises econômicas que enfocam custos de tratamentos ${ }^{(5)}$.

\section{TIPOS DE MEDIDAS DE SAÚDE}

Na literatura, as medidas do estado de saúde são abordadas como medidas de utilidade, instrumentos genéricos e específicos ${ }^{(3,5)}$.

\section{MEDIDAS DE UTILIDADE}

As medidas de utilidade capturam valores gerais do estado de saúde através da avaliação da preferência individual para um estado sobre o outro. Na prática clínica, facilitam tomadas de decisão, determinando preferências num cenário clínico com diversas possibilidades de resultados de tratamento. Esse tipo de medida é fundamental em análise econômica do tipo custo-utilidade $^{(3,5)}$. Especialidades cirúrgicas são bem representadas por esse tipo de medida quando se tem mais de uma opção de tratamento para uma determinada enfermidade ${ }^{(6)}$. Na comunidade ortopédica existem poucos estudos de análise de decisão e uso de medidas de utilidade ${ }^{(6)}$. Sporer e Rosenberg, em estudo de revisão sobre análise de decisão e ortopedia, criaram um cenário clínico para tratamento de fratura exposta de tíbia Gustilo III-C - amputação versus salvamento do membro ${ }^{(6)}$. Tosteon revisou as medidas de utilidade aplicadas à lombalgia ${ }^{(7)}$. As medidas de utilidade mais comumente utilizadas na literatura são o Euroqol (EQ-5D) e o Health Utility Index ${ }^{(3)}$.

\section{INSTRUMENTOS GENÉRICOS}

Os instrumentos classificados como genéricos quantificam a percepção do paciente sobre seu estado de saúde geral. Sua desvantagem é o fato de que podem não detectar mudanças em aspectos específicos ${ }^{(3,5)}$. Apesar disso, eles são suficientemente precisos para análises de grupos sobre o aspecto geral de saúde. Dessa forma, permitem comparações de um grupo com um modelo populacional ou entre diferentes enfermidades. A maioria deles tem mais de um domínio e discrimina funções físicas, emocionais e sociais ${ }^{(3)}$. $\mathrm{O}$ instrumento genérico mais comumente utilizado na literatura ortopédica é o Short-Form 36 (SF-36) ${ }^{(3-4)}$. Seu uso tem sido recomendado associado ao de instrumentos específicos em estudos de prosseguimento das desordens músculo-esqueléticas ${ }^{(4)}$.

\section{INSTRUMENTOS ESPECÍFICOS}

A literatura descreve dois tipos de instrumentos específicos: aqueles para determinada extremidade ou articulação e os específicos para doenças. Os específicos para extremidade oferecem uma alternativa prática, já que podem ser usados num contexto de várias enfermidades ${ }^{(3)}$. Entretanto, o instrumento deve adequar suas propriedades psicométricas (confiabilidade, validade e responsividade) em todos os grupos de pacientes em que é aplicado. Como exemplos, existem o Short Musculoskeletal Functional Assessment (SMFA) ${ }^{(8)}$, desenvolvido para avaliar qualquer desordem músculo-esquelética da extremidade superior ou inferior, e o Disabilities of the Arm, Shoulder and Hand questionnaire (DASH) ${ }^{(9)}$ para avaliação do membro superior.

Os instrumentos específicos para determinada articulação ou doença são os mais comuns e com maior variedade na literatura ortopédica. Alguns autores criticam essa variedade por dificultar comparações de resultados entre diferentes estudos ${ }^{(3,10)}$. Entretanto, a maior vantagem desse tipo de medida é a habilidade de detectar mudanças específicas de determinadas 
doenças quando estas ocorrem ${ }^{(3,5)}$. Os questionários Western Ontario Rotator Cuff Index e Western Ontario Shoulder Instability Index, ambos desenvolvidos pelo Kirkley Research Group, são exemplos de instrumentos específicos ${ }^{(11-12)}$. Os mesmos abordam o impacto de certas atividades ou posições específicas nas desordens do manguito rotador e nas instabilidade de ombro, respectivamente. Um paciente que, por exemplo, tenha evitado tais posições poderia reportar boa função através de outro instrumento de ombro que não trate dessas possíveis alterações específicas. Portanto, se o objetivo de um estudo é discernir resultados entre dois tipos de tratamento em alguma enfermidade, o instrumento específico poderia ser a melhor forma de detectar diferenças ${ }^{(3)}$.

A maioria dos questionários usados na área de ortopedia foi desenvolvida na língua inglesa. Quando já existe alguma medida adequadamente validada em outro idioma que avalie a condição de interesse, torna-se desnecessário o desenvolvimento de um novo instrumento, por demandar tempo e custo maiores ${ }^{(13)}$. Sendo assim, foi padronizada a metodologia de equivalência transcultural ${ }^{(14-16)}$. Esse processo consiste em realizar a tradução, adaptação cultural e validação do instrumento proposto. As etapas de tradução e adaptação cultural permitem ajustar o instrumento ao novo idioma, população, contexto e cultura. A fase de validação consiste em verificar se o novo instrumento manteve as características da versão original. As propriedades psicométricas mais comumente analisadas nessa fase são validade, reprodutibilidade e responsividade. Todo esse processo é relevante para que o instrumento seja culturalmente aceito no país e equivalente à sua versão original ${ }^{(1,13-16)}$.

As propriedades psicométricas de muitos dos instrumentos utilizados na literatura ortopédica não foram citadas ou não seguem os padrões recomendados para seu desenvolvimento. A variedade de instrumentos disponíveis na literatura e as limitações dos estudos dificultam a escolha de um questionário que siga os critérios mínimos ou que garanta uma avaliação adequada para determinada população. Dessa forma, numerosos estudos têm analisado criteriosamente os instrumentos disponíveis, avaliado suas propriedades e publicado recomendações para conceitualizar, desenvolver, avaliar e comparar tais instrumentos, direcionando a escolha dos mais adequados para serem usados pela comunidade ortopédica ${ }^{(3,10,17-23)}$.

Beaton et al avaliaram o conteúdo de seis periódicos da área de ortopedia e discriminaram os instrumentos mais comumente utilizados e suas propriedades psicométricas ${ }^{(3)}$. De acordo com seus resultados, o instrumento mais usado para avaliar a percepção do paciente foi o SF-36. Outras medidas destacadas foram os instrumentos regionais $\mathrm{SMFA}^{(8)} \mathrm{e}$ o DASH${ }^{(9)}$. Os questionários específicos mais comumente encontrados na literatura foram o Rolland Morris Questionnaire ${ }^{(24)}$ para lombalgia, seguido do Master Ostheoarthritis Index (WOMAC) ${ }^{(25)}$ para osteoartrose de joelho e quadril.

\section{INSTRUMENTOS DISPONÍVEIS NO BRASIL}

Reconhecendo a importância do uso de medidas do estado de saúde para avaliação de intervenções e de novas técnicas, estudos de equivalência cultural e validação dos principais instrumentos utilizados na literatura ortopédica internacional têm sido desenvolvidos no Brasil seguindo os critérios recomendados. $\mathrm{O}$ quadro 1 cita alguns dos instrumentos disponíveis para o uso.

\section{CONCLUSÃO}

A variedade de instrumentos disponíveis na literatura ortopédica dificulta análises de correlação de estudos. Para que a comunidade ortopédica possa utilizar essas variáveis para demonstrar benefícios de novas técnicas ou comparar tipos de tratamentos, é necessária a uniformidade do uso dos instrumentos adequados para cada articulação ou doença, associado à utilização de um instrumento genérico. 
QUADRO 1

Instrumentos disponíveis para uso no Brasil

\begin{tabular}{|c|c|c|}
\hline Instrumento & Classificação & O que avalia \\
\hline Short-Form 36 (SF-36) $^{(26)}$ & Genérico & Qualidade de vida relacionada à saúde \\
\hline $\begin{array}{l}\text { Nottingham Health } \\
\text { Profile(14) }^{(14)}\end{array}$ & Genérico & Qualidade de vida \\
\hline $\begin{array}{l}\text { Short Musculoskeletal } \\
\text { Function Assessment } \\
\text { (SMFA)(27) }^{(S M F}{ }^{(27)}\end{array}$ & $\begin{array}{l}\text { Específico - } \\
\text { extremidades }\end{array}$ & $\begin{array}{l}\text { Função músculo-esquelética nos } \\
\text { membros superiores e inferiores }\end{array}$ \\
\hline $\begin{array}{l}\text { Disabilities of Arm, } \\
\text { Shoulder and Hand } \\
(\mathrm{DASH})^{(28)}\end{array}$ & $\begin{array}{l}\text { Específico - } \\
\text { extremidades }\end{array}$ & $\begin{array}{l}\text { Capacidade funcional nas } \\
\text { enfermidades do membro superior }\end{array}$ \\
\hline $\begin{array}{l}\text { Western Ontario Rotator } \\
\text { Cuff Index (WORC) })^{(1)}\end{array}$ & $\begin{array}{l}\text { Específico - } \\
\text { doença }\end{array}$ & $\begin{array}{l}\text { Qualidade de vida nas enfermidades } \\
\text { do manguito rotador }\end{array}$ \\
\hline $\begin{array}{l}\text { Athletic Shoulder } \\
\text { Outcome Rating Scale }\end{array}$ & $\begin{array}{l}\text { Específico - } \\
\text { articulação }\end{array}$ & $\begin{array}{l}\text { Escala para avaliação das afecções do } \\
\text { ombro em atletas }\end{array}$ \\
\hline $\begin{array}{l}\text { Oswestry Disabilty } \\
\text { Index }\end{array}$ & $\begin{array}{l}\text { Específico - } \\
\text { doença }\end{array}$ & $\begin{array}{l}\text { Capacidade funcional em pacientes } \\
\text { com lombalgia }\end{array}$ \\
\hline $\begin{array}{l}\text { Rolland Morris } \\
\text { Questionnaire }\end{array}$ & $\begin{array}{l}\text { Específico - } \\
\text { doença }\end{array}$ & $\begin{array}{l}\text { Capacidade funcional em pacientes } \\
\text { com lombalgia }\end{array}$ \\
\hline $\begin{array}{l}\text { Western Ontario } \\
\text { McMaster Arthritis } \\
\text { Index (WOMAC) }{ }^{(32)}\end{array}$ & $\begin{array}{l}\text { Específico - } \\
\text { articulação }\end{array}$ & $\begin{array}{l}\text { Avaliação funcional em osteoartrose } \\
\text { de quadril e joelho }\end{array}$ \\
\hline Lysholm Scale ${ }^{(33)}$ & $\begin{array}{l}\text { Específico - } \\
\text { doença }\end{array}$ & $\begin{array}{l}\text { Avaliação de enfermidades } \\
\text { ligamentares de joelho }\end{array}$ \\
\hline $\begin{array}{l}\text { American Orthopaedic } \\
\text { Foot and Ankle Society } \\
\text { Ankle-Hindfoot Scale } \\
(A O F A S)^{(34)}\end{array}$ & $\begin{array}{l}\text { Específico - } \\
\text { articulação }\end{array}$ & $\begin{array}{l}\text { Escala de avaliação para } \\
\text { tornozelo e pé }\end{array}$ \\
\hline
\end{tabular}

\section{REFERÊNCIAS}

1. Lopes AD, Stadniky SP, Masiero D, Carrera EF, Ciconelli RM, Griffin S. Tradução e adaptação cultural do WORC: um questionário de qualidade de vida para alterações do manguito rotador. Rev Bras Fisioter. 2006;10(3):309-15.

2. Kirkley A, Griffin S. Development of disease-specific quality of life measurement tools. Arthroscopy. 2003;19(10):1121-8.

3. Beaton DE, Schemitsch E. Measures of health-related quality of life and physical function. Clin Orthop Relat Res. 2003; (413):90-105.

4. Gay RE, Amadio PC, Johnson JC. Comparative responsiveness of the disabilities of the arm, shoulder, and hand, the carpal tunnel questionnaire, and the SF-36 to clinical change after carpal tunnel release. J Hand Surg [Am]. 2003;28(2):250-4.

5. Campolina AG, Ciconelli RM. Qualidade de vida e medidas de utilidade: parâmetros clínicos para as tomadas de decisão em saúde. Rev Panam Salud Pública = Pan Am J Public Health. 2006;19(2):128-36.
6. Sporer SM, Rosenberg AG. Decision analysis in orthopaedics. Clin Orthop Relat Res. 2005;(431):250-6.

7. Tosteson AN. Preference-based health outcome measures in low back pain. Spine. 2000;25(24):3161-6.

8. Martin DP, Engelberg R, Agel J, Snapp D, Swiontkowski MF. Development of a musculoskeletal extremity health status instrument: the Musculoskeletal Function Assessment instrument. J Orthop Res. 1996;14(2):173-81.

9. Beaton DE, Katz JN, Fossel AH, Wright JG, Tarasuk V, Bombardier C. Measuring the whole or the parts? Validity, reliability, and responsiveness of the Disabilities of the Arm, Shoulder and Hand outcome measure in different regions of the upper extremity. J Hand Ther. 2001;14(2):128-46.

10. Weitzel PP, Richmond JC. Critical evaluation of different scoring systems of the knee. Sports Med Arthrosc Rev. 2002; 10(3):183-90. 
11. Kirkley A, Alvarez C, Griffin S. The development and evaluation of a disease-specific quality-of-life questionnaire for disorders of the rotator cuff: The Western Ontario Rotator Cuff Index. Clin J Sport Med. 2003;13(2):84-92.

12. Kirkley A, Griffin S, McLintock H, Ng L. The development and evaluation of a disease-specific quality of life measurement tool for shoulder instability. The Western Ontario Shoulder Instability Index (WOSI). Am J Sports Med. 1998;26(6):76472.

13. Teixeira-Salmela LF, Magalhaes LC, Souza AC, Lima MC, Lima RCM, Goulart F. Adaptação do Perfil de Saúde de Nottingham: um instrumento simples de avaliação da qualidade de vida. Cad Saúde Pública = Rep Public Health. 2004;20(4):905-14.

14. Herdman M, Fox-Rushby J, Badia X. 'Equivalence' and the translation and adaptation of health-related quality of life questionnaires. Qual Life Res. 1997;6(3):237-47.

15. Guillemin F. Cross-cultural adaptation and validation of health status measures. Scand J Rheumatol. 1995;24(2):61-3. Comment on: Scand J Rheumatol. 1995;24(2):64-8. Scand J Rheumatol. 1995;24(2):69-75.

16. Guillemin F, Bombardier C, Beaton D. Cross-cultural adaptation of health-related quality of life measures: literature review and proposed guidelines. J Clin Epidemiol. 1993; 46(12):1417-32. Comment in: J Clin Epidemiol. 1994;47(12): 1465-6.

17. Dowrick AS, Gabbe BJ, Williamson OD, Cameron PA. Outcome instruments for the assessment of the upper extremity following trauma: a review. Injury. 2005;36(4):468-76.

18. Agel J, Swiontkowski MF. Guide to outcomes instruments for musculoskeletal trauma research. J Orthop Trauma. 2006;20(8 Suppl): S1-146.

19. Müller U, Röder C, Greenough CG. Back related outcome assessment instruments. Eur Spine J. 2006;15 Suppl 1:S25-31.

20. Grotle M, Brox JI, Vollestad NK. Functional status and disability questionnaires: what do they assess? A systematic review of back-specific outcome questionnaires. Spine. 2005; 30(1):130-40.

21. Bot SD, Terwee CB, van der Windt DA, Bouter LM, Dekker J, de Vet HC. Clinimetric evaluation of shoulder disability questionnaires: a systematic review of the literature. Ann Rheum Dis. 2004;63(4):335-41.

22. Kirkley A, Griffin S, Dainty K. Scoring systems for the functional assessment of the shoulder. Arthroscopy. 2003; 19(10):1109-20.

23. Button G, Pinney S. A meta-analysis of outcome rating scales in foot and ankle surgery: is there a valid, reliable, and responsive system? Foot Ankle Int. 2004;25(8):521-5.
24. Roland M, Morris R. A study of the natural history of back pain. Part I: development of a reliable and sensitive measure of disability in low-back pain. Spine. 1983;8(2):141-4.

25. Bellamy N, Buchanan WW, Goldsmith CH, Campbell J, Stitt LW. Validation study of WOMAC: a health status instrument for measuring clinically important patient relevant outcomes to antirheumatic drug therapy in patients with osteoarthritis of the hip or knee. J Rheumatol. 1988;15(12):1833-40.

26. Ciconelli RM, Ferraz MB, Santos W, Meinão I, Quaresma MR. Tradução para a língua portuguesa e validação do questionário genérico de avaliação de qualidade de vida SF-36 (Brasil SF36). Rev Bras Reumatol. 1999;39(3):143-50.

27. Taylor MK, Pietrobon R, Menezes A, Olson SA, Pan D, Bathia $\mathrm{N}$, et al. Cross-cultural adaptation and validation of the Brazilian Portuguese version of the short musculoskeletal function assessment questionnaire: the SMFA-BR. J Bone Joint Surg Am. 2005;87(4):788-94.

28. Orfale AG, Araújo PM, Ferraz MB, Natour J. Translation into Brazilian Portuguese, cultural adaptation and evaluation of the reliability of the Disabilities of the Rrm, Shoulder and Hand Questionnaire. Braz J Med Biol Res. 2005;38(2):293-302.

29. Leme L. Tradução, adaptação cultural e validação da escala "Athletic Shoulder Outcome Rating Scale" para a língua portuguesa [tese]. São Paulo: Escola Paulista de Medicina, Universidade Federal de São Paulo; 2005.

30. Vigatto R, Alexandre NM, Correa Filho HR. Development of a Brazilian Portuguese version of the Oswestry Disability Index: cross-cultural adaptation, reliability, and validity. Spine. 2007; 32(4):481-6.

31. Nusbaum L, Natour J, Ferraz MB, Goldenberg J. Translation, adaptation and validation of the Roland-Morris questionnaire Brazil Roland-Morris. Braz J Med Biol Res. 2001;34(2):20310.

32. Fernandes MI. Tradução e validação do questionário de qualidade de vida específico para osteoartrose WOMAC (Western Ontario McMaster Universities) para a língua portuguesa [tese]. São Paulo: Escola Paulista de Medicina, Universidade Federal de São Paulo; 2003.

33. Peccin MS, Ciconelli R, Cohen M. Questionário específico para sintomas do joelho "Lysholm Knee Scoring Scale": tradução e validação para a língua portuguesa. Acta Ortop Bras. 2006;14(5): 268-72.

34. Rodrigues RC. Tradução, adaptação cultural e validação para língua portugues ao "American Orthopaedic Foot and Ankle Society (AOFAS) Ankle-Hindfoot Scale" [tese]. São Paulo: Escola Paulista de Medicina, Universidade Federal de São Paulo; 2005 . 